\title{
The Human Tail: A Simple Skin Appendage or Cutaneous Stigma of an Anomaly?
}

\author{
Cezmi Cagri TURK ${ }^{1}$, Niyazi Nefi KARA ${ }^{1}$, Ali BACANLI²
}

${ }^{1}$ Antalya Education and Research Hospital, Department of Neurosurgery, Antalya Turkey

${ }^{2}$ Antalya Education and Research Hospital, Department of Dermatology, Antalya Turkey

Presented in: $27^{\text {th }}$ Scientific Congress of Turkish Neurosurgical Society, April 12-16, 2013, Antalya, Turkey

\section{ABSTRACT}

AIM: The human tail is a term to describe skin-covered protrusions in the lumbosacral and coccygeal region, ascribing to the resemblance to the tails in the animals. The first reports dates back to second half of the $19^{\text {th }}$ century, the etiological bases could not been ascertained yet. They are mainly classified as true or pseudo-tails.

MATERIAL and METHODS: Five cases with human tails were diagnosed and managed in our hospital between 2010 and 2014. Their demographic and lesion characteristics are presented.

RESULTS: Three of the 5 cases were male patients. The ages ranged between 1 day and 50 years at the time of diagnosis. The patients were diagnosed basically by the external appearance of the lesions without neurological deficits. Detailed examination revealed several associated lesions: two dermal sinus tracts, one tethered spinal cord and one club-foot in one-day preemie. Two patients had true and 3 had pseudo-tails. Four of them underwent surgery but the last one did not accept surgery. Surgery consisted of simple excision of the lesion in 2 patients with true tails and excision and removal of dermal sinus tract and untethering when necessary in the other 2 pseudo-tails.

CONCLUSION: The presented study indicated that true human tails are simple skin appendages without any associated spinal anomalies. However, pseudo-tails are potentially complex lesions with a high risk of spinal dysraphisms; warranting further diagnostic work-up and more extensive surgical technique if necessary. The key to managing human tails is making a clear distinction between true tails and pseudo-tails.

KEYWORDS: Classification, Human tail, Spinal dysraphism, Surgery

\section{INTRODUCTION}

The human tail is a term to describe skin-covered protrusions in the lumbosacral and coccygeal region, ascribing to the resemblance to the tails in the animals. The first reports date back to the second half of the 19th century (3), however, the etiological basis has not been ascertained yet. Dao and Netsky et al. compiled and classified these lesions in 33 patients, denoting these lesions as true or pseudo-tails (3). Although this classification is under debate and criticized for insufficient clinical application, it still maintains its place in the centre of the reports.

An advance in imaging modalities has affected the approach to these lesions in defining the nature and clinical significance by detecting associated anomalies and shaping treatment strategies (4).

In the following report, five patients with human tails are presented and the clinical significance is discussed based on contemporary literature data. 


\section{MATERIAL and METHODS}

Five cases with human tails were diagnosed and managed in our hospital between 2010 and 2014. Three of the 5 cases were male. Their ages ranged between 1 day and 50 years at the time of diagnosis. All patients except the adult patient were referred by obstetricians, pediatric surgeons or dermatologists. The adult patient was first evaluated by general surgeons and neurosurgical consultation was advised because of the location and extension of the lesion. There was generally no associated neurological deficits at the time of diagnosis in our patients. They were referred basically for the external appearance of the lesions. However, detailed examination revealed dermal sinus tracts and club-foot in the one-day preemie. Magnetic resonance (MR) images were obtained for probable spinal dysraphisms (Table I). Two of the cases were classified as true human tails, and the others as pseudo-tails. Four of the cases in childhood were operated; however, the adult patient did not accept the surgery.

\section{RESULTS}

\section{Example Cases}

Case number 1: A one-month-old male patient was referred by a pediatric surgeon for the consultation of a tail-like skin protrusion from sacrococcygeal region. His neurological examination was not striking. His MR scans demonstrated fusion defects in the lamina, extension of the appendage into the spinal canal and spinal cord tethering, ending at the L3-4 level (Figure 1A-D). Histopathological evaluation revealed mature epidermal and dermal linings (Figure 2).

Case number 3: A three-years old male patient was referred by a pediatric surgeon for a tail-like protrusion from sacral region without any associated anomalies. The family admitted the lesion was diagnosed after birth and surgical removal had been recommended several months later but they were lost to follow-up. Soon after the patient was admitted, he was operated and the skin appendage was removed without any complication (Figure 3A, B). Histopathological evaluation demonstrated tail-like protrusion with mature epidermal and dermal linings (Figure 4).

Case number 5: A fifty-year-old male patient was referred by general surgeons for evaluation of a sacrococcygeal mass lesion. The patient's history revealed the presence of a skin appendage, which was diagnosed soon after the birth but could not be removed due to his parents' unwillingness. As the patient did not feel any discomfort other than cosmesis, the lesion was left untouched until last year. Then, the patient reported falling from a chair on his buttock which did not cause any obvious complications at that time. However, the size of the appendage increased in the last year and started to fluctuate upon squeezing and sitting. It was though that there was a connection to the subarachnoid space through coccygeal region. His MR scans were suspicious for a connection through the coccygeal region with obvious collection in the subcutaneous region extending to the coccygeal level. He was advised to undergo surgical treatment but he did not consent to surgery (Figure 5A-D).

\section{DISCUSSION}

The human tail is a rare congenital anomaly accompanying many medical obscurities. Unfamiliarity arises concerns about the prognosis and the best management strategies, besides familial and social concerns (6). It has been reported at different lengths and at different parts of the spine; from the coccyx to the lumbar region or arising from the midline to the paravertebral areas with different clinical attributes, significance and classification schemes (4).

\section{Classification}

The classification by Dao and Netsky in 1984 is generally

Table I: Demographic and Lesion Characteristics of the Patients with Human Tails

\begin{tabular}{|c|c|c|c|c|c|c|c|}
\hline Case & AgelSex & Complaint & $\begin{array}{l}\text { Neurological } \\
\text { Exam }\end{array}$ & Level & $\begin{array}{l}\text { Associated } \\
\text { Anomaly }\end{array}$ & Type & $\begin{array}{l}\text { Treatment } \backslash \\
\text { Prognosis }\end{array}$ \\
\hline 1 & $\begin{array}{l}1 \text { month } \\
\text { Male }\end{array}$ & Tail-like appendage & Normal & Lumbosacral & $\begin{array}{l}\text { Tethered cord } \\
\text { and Dermal } \\
\text { sinus tract }\end{array}$ & Pseudo & $\begin{array}{l}\text { Surgical excision, } \\
\text { untethering and } \\
\text { dermal sinus tract } \\
\text { removal }\end{array}$ \\
\hline 2 & $\begin{array}{l}3 \text { month } \\
\text { Female }\end{array}$ & $\begin{array}{l}\text { Protruding lesion in } \\
\text { intergluteal area }\end{array}$ & Normal & Sacrococcygeal & None & True & Surgical excision \\
\hline 3 & $\begin{array}{l}3 \text { years } \backslash \\
\text { Male }\end{array}$ & Tail-like appendage & Normal & Sacral & None & True & Surgical excision \\
\hline 4 & $\begin{array}{l}1 \text { day } \\
\text { Female }\end{array}$ & $\begin{array}{l}\text { Protrusion in } \\
\text { intergluteal region } \\
\text { and dermal sinus } \\
\text { tracts }\end{array}$ & Club Foot & Sacral & $\begin{array}{l}\text { Dermal sinus } \\
\text { tract }\end{array}$ & Pseudo & $\begin{array}{l}\text { Surgical excision } \\
\text { and dermal sinus } \\
\text { tracts removal }\end{array}$ \\
\hline 5 & $\begin{array}{l}50 \text { years } \backslash \\
\text { Male }\end{array}$ & $\begin{array}{l}\text { Protruding lesion in } \\
\text { sacral region }\end{array}$ & Normal & Sacrococcygeal & None & Pseudo & Follow-up \\
\hline
\end{tabular}




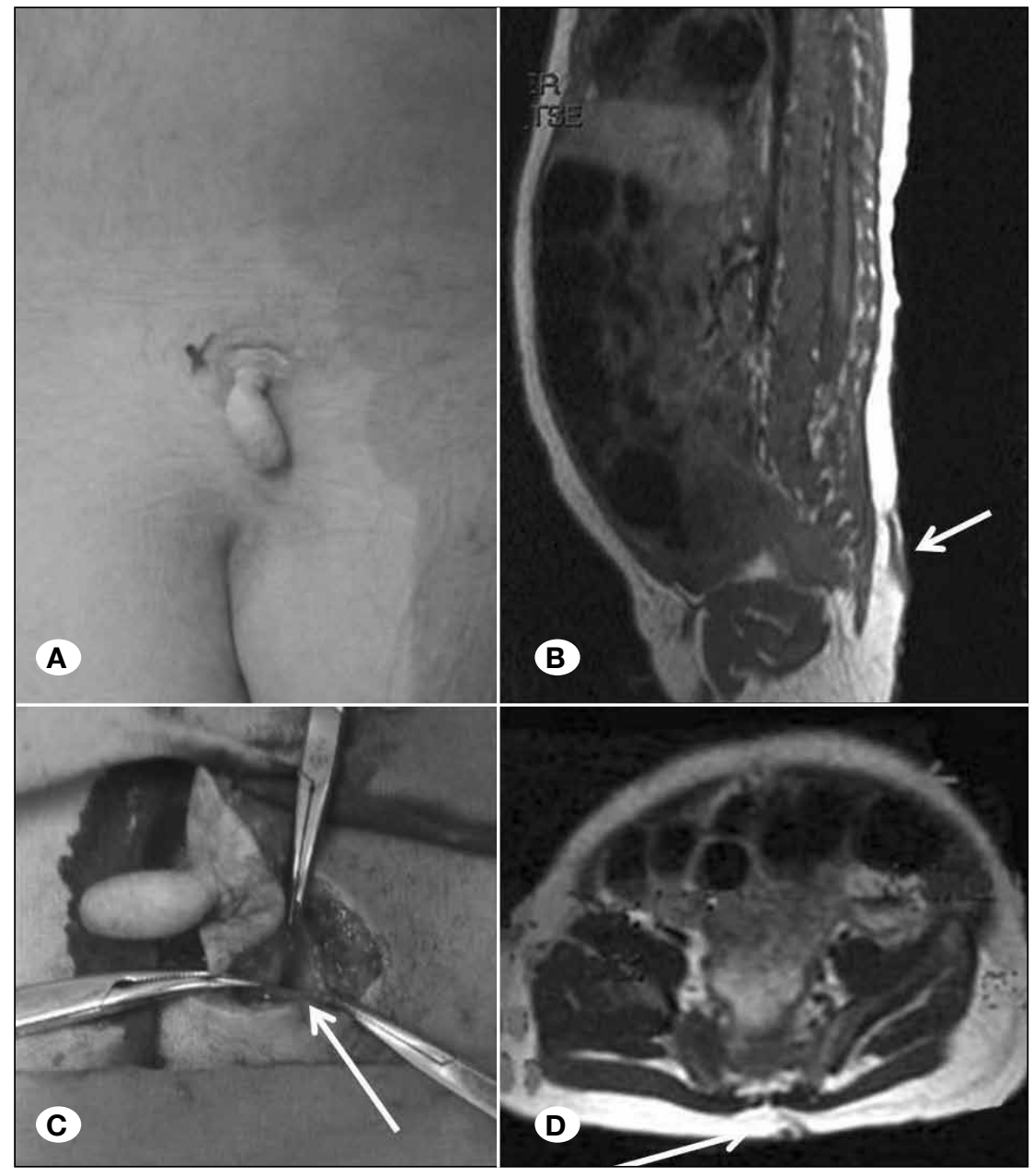

Figure 1: A) Picture of pseudo-human tail (case 1) in the lumbosacral region, $\mathbf{B}$ and $\mathbf{D}$ ) sagital and axial MR scans; arrow in (B) indicating the tail, arrow in (D) indicating the dermal sinus tract extending to the spinal canal. C) shows the intraoperative view, the human tail was excised circumferentially and the opening of the dermal sinus is demonstrated (arrow).

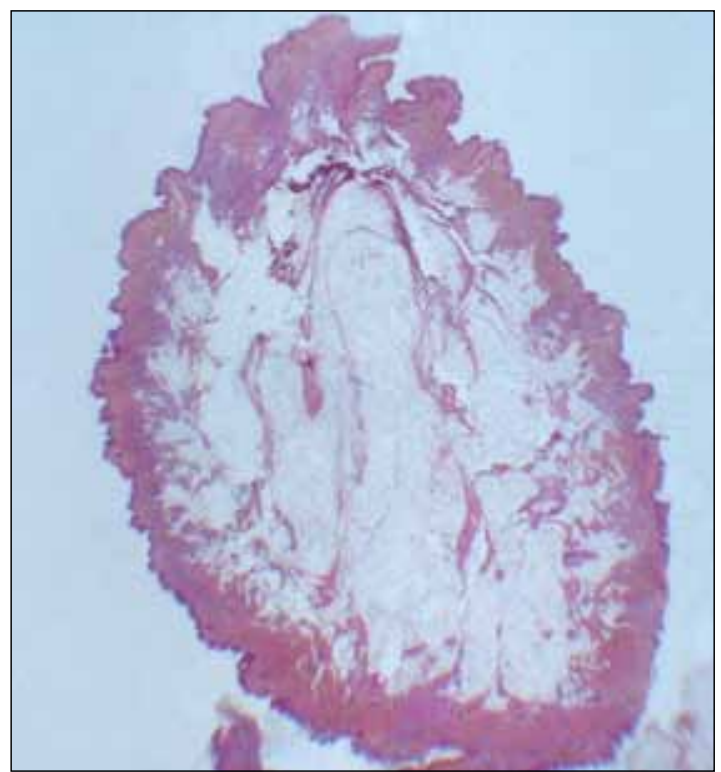

Figure 2: Hematoxylin and eosin staining of the lesion demonstrating mature epidermal and dermal linings with mild papillomatosis. The subcutaneous region shows increased number of mature adipocytes (H\&E stain X6). referred to for classification of human tails as true or pseudo tail (3). In spite of its connotations, true, in this classification, did not imply close resemblance of the protrusion to a tail, per se, instead; to a structure that may be composed of fatty and connective tissues, including muscle structures and blood vessels without any connection to the spinal canal (3). It is thought to be a benign entity as there is no connection to neural tissues. On the other hand, the pseudo tail term includes those lesions protruding and located in the lumbosacral region and demonstrating resemblance to persistent vestigial tails (true tails) superficially such as coccygeal vertebrae, lipomas, teratomas, chondromegaly, glioma and elongated parasitic fetus (3).

Recent reports have criticized this classification for being inconclusive and obscure from a clinical perspective and naming almost all cases as pseudo-tails. Moreover, it was claimed that this classification was not supported by embryological findings (9). The authors of the report also share similar thoughts as the classification is neither a useful tool for clinical application nor provides clear etiological understanding.

Lin et al. provided a classification scheme based on anatomic location and presence of associated lesions (spinal dys- 


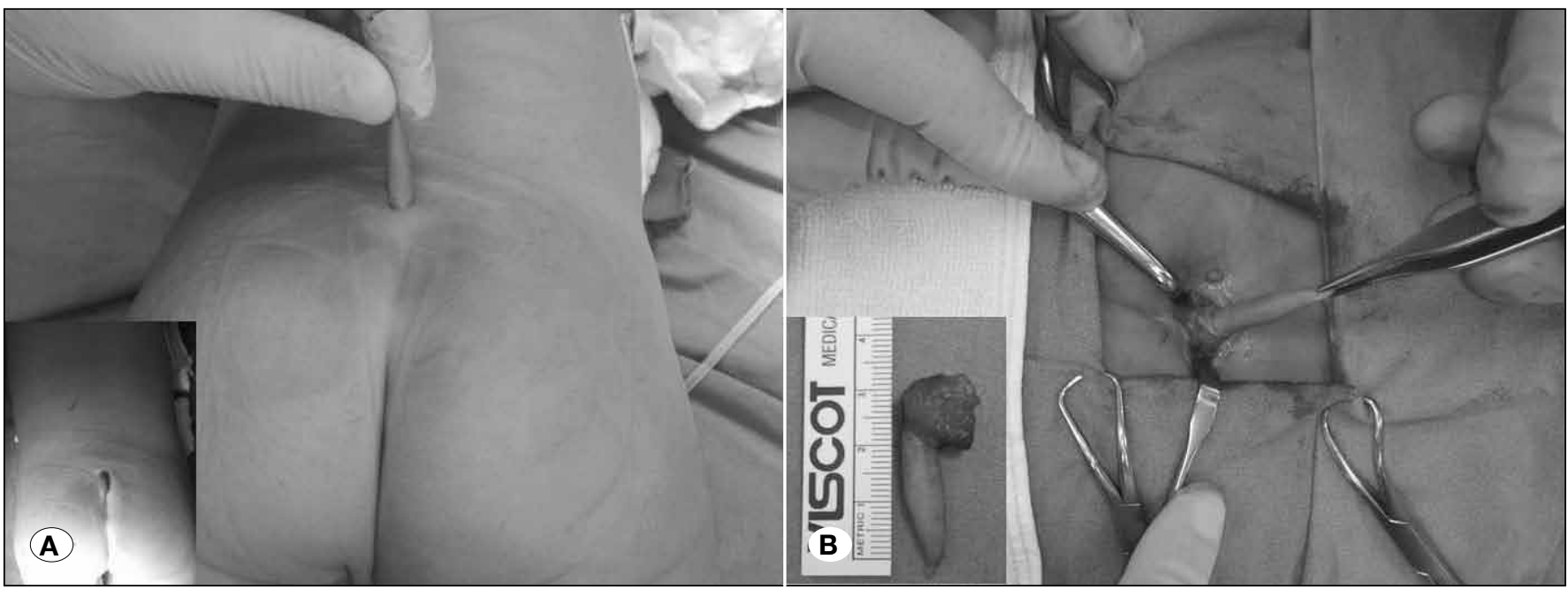

Figure 3: A) A true tail in Case no: 3 B) intraoperative view showing excision of the tail.

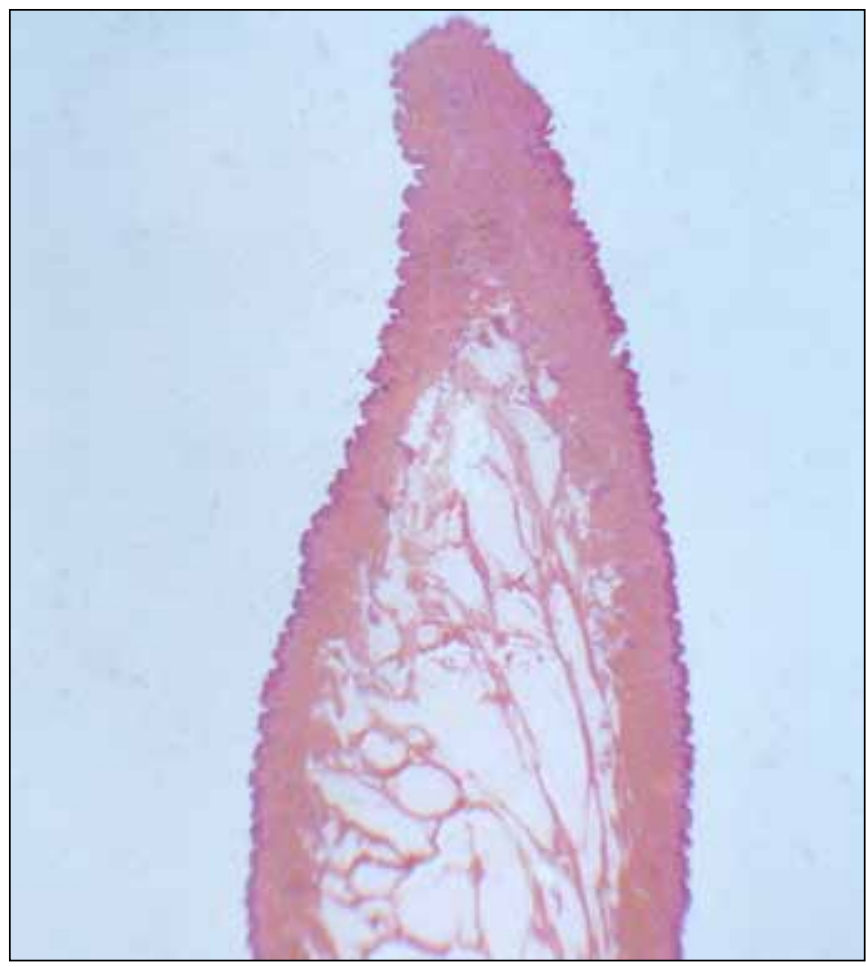

Figure 4: A tail-like appendage with normally appearing cutaneous structures and mild papillomatosis with increase in mature adipose tissue in the subcutaneous region (H\&E stain X 6).

raphism, lipoma, tethered cord). According to this classification, a lesion located in lumbosacral region is a pseudo-tail, whether associated with spinal dysraphism or not. However, if the same lesion is situated in the gluteal or coccygeal region, association with other lesions is important for deciding on the diagnosis; it is a pseudo-tail if there are any accompanying lesions and it is called a true tail if not (8). Similarly, Cai et al. also considered true tails as benign and cosmetic lesions while pseudo-tails were accepted as a more complex events, repre- senting a cutaneous marker for underlying spinal dysraphism, and detailed diagnostic examinations were recommended (2).

\section{Associated Lesions}

Human tails may be associated with other developmental and non-developmental conditions like spinal dysraphisms, spina bidifa, tethered cord, coccygeal vertabrae, syndactly, and lipomas. There are also few familial cases that were reported previously $(1,9)$. In an article on lumbosacral mass lesions, the series included lipomas (67\%), teratomas (25\%) and meningocele cases (4\%) (10). It was reported that spinal dysraphism, mostly spina bifida and meningocele, were associated with human tails in $49 \%$ of the patients. The figures were $27 \%$ and $20 \%$ for lipomas and tethered cord, respectively. Club foot was reported in 1 patient in a series of 59 patients (9). In our series, we had one patient with clubfoot anomaly. Two of our patients had associated dermal sinus tracts, only one having tethered spinal cord.

Based on these data, human tails should not be classified as benign lesions straightforwardly in spite of some previous reports (12), and they should undergo more detailed radiological work-up because of the risk of underlying spinal cord involvement.

\section{Surgical Treatment}

Management of human tails is basically surgical (5), however, what differs is the extent of surgery depending on the lesion being a true tail or a pseudo-tail. A true human tail is accepted as a failure of regression in fetal life and so this caudal appendage should be treated as a benign lesion and the treatment is simple excision for cosmetic reasons. On the other hand, a pseudo-tail is generally a cutaneous representation of an underlying associated condition and has a common ectodermal origin with the skin and nervous system. Surgical treatment will include more extensive surgery for both removal of the lesion and untethering of the spinal cord or removing dermal sinus tracts, when necessary (8). In our series, 3 cases had pseudo-tails and other 2 had true tails. All of the 


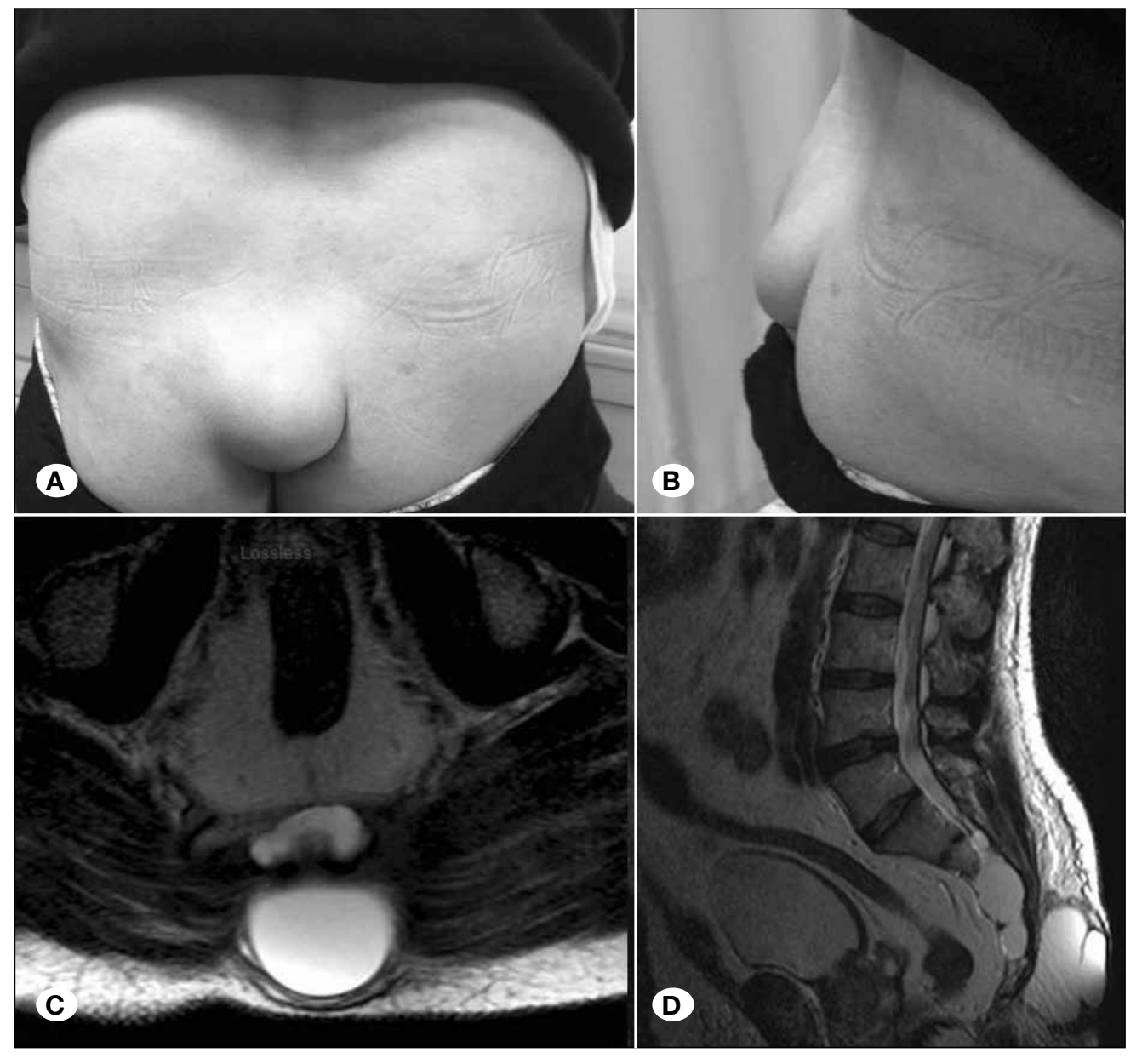

Figure 5: A, B) Posterior and lateral pictures of case no: 5 demonstrating the lesion in the intergluteal region, C, D) axial and sagital MR scans showing CSF collection beneath the lesion with suspected connection to the spinal canal through the coccygeal region. true lesions were treated with simple surgical excision of the tail. Two patients with pseudo-tails were operated for deeper extensions and removal of dermal sinus tracts. Untethering of the spinal cord was only performed in one case. The surgical procedure was uncomplicated in all 4 patients.

Spinal dysraphism therefore needs to be sought and the distinction between a true and pseudo-tail should be made in every case $(5,7)$.

One other question about the surgery may be the timing. In the literature one article address this issue and indicates that the timing was basically related to the presence of a tethered cord. If neurological findings allow, delaying the surgery for 3 months or until the patients' weight reaches $5 \mathrm{~kg}$ has been recommended (11). In the literature, one other report emphasizes the probable deterioration in years with a tethered cord, and suggests early surgery for untethering before 2 years of age in order to keep the patients free of symptoms. Moreover, long-term follow-up was recommended (9).

\section{Prognosis}

The natural course of the human tail has not been well reported previously. Donovan and Pedersen et al. showed about 50\% growth of the tail in the first month, involving both the length and the thickness (4). Moreover, they postulated probable disappearance of dermal sinus tracts that were present at birth, but lost at follow-up MRs. In our series, we observed the late stages of human tails; one patient who was 3 years old was diagnosed at birth but lost to follow up to another center and observed 3 years later in our clinic. The family claimed elongation of the tail. However, we do not have any objective measurement. The other patient was unique in that the patient's presentation was in adulthood. The lesion was claimed to be finger-like at birth. No growth in the lesion was observed before the falling incident. There could have been some growth beyond the memory of the patient in the childhood period, however, this information is obscure at the moment. It is still interesting that the lesion enlarged gradually following the falling incident, which might have caused recanalisation of the dermal sinus tracts or a rudimentary connection to the spinal canal leading to dilation of the skin-appendage in the gluteal region. The natural course of pseudo-tails need further reports to elucidate the potential for any complications in the late period.

\section{CONCLUSIONS}

The presented study showed that true human tails are simple skin appendages without any associated spinal anomalies and have a favorable outcome. However, pseudo-tails are 
potentially complex lesions with risk of spinal dysraphism, tethered cord, and dermal sinus tracts warranting further diagnostic work-up and extended surgical technique. The key to managing human tails is making a clear distinction between true tails and pseudo-tails with familiarity with the lesion and vigilance.

\section{- REFERENCES}

1. Akhil P, Ashutosh N, Fais F, Shashank M, Sanjay P, Singhal BM, Attri PC, Arvind G: True vestigeal tail with lumbosacral meningomyelocoel: A rare case report. $\mathrm{Br} \mathrm{J}$ Neurosurg 24:597599, 2010

2. Cai C, Shi O, Shen C: Surgical treatment of a patient with human tail and multiple abnormalities of the spinal cord and column. Adv Orthop 2011:153797, 2011

3. Dao AH, Netsky MG: Human tails and pseudotails. Hum Pathol 15:449-453, 1984

4. Donovan DJ, Pedersen RC: Human tail with noncontiguous intraspinal lipoma and spinal cord tethering: Case report and embryologic discussion. Pediatr Neurosurg 41:35-40, 2005
5. Ersahin Y, Gezen F, Baysefer A, Beduk A: Neuroectodermal appendage: A case report and review. Turk Neurosurg 3:25-27, 1993

6. Kumar D, Kapoor A: Human tail: Nature's aberration. J Child Neurol 27:924-926, 2012

7. Ledley FD: Evolution and the human tail. $\mathrm{N}$ Engl $\mathrm{J}$ Med 306 (20):1212-1215, 1982

8. Lin PJ, Chang YT, Tseng HI, Lin JY, Huang YS: Human tail and myelomeningocele. Pediatr Neurosurg 43:334-337, 2007

9. Lu FL, Wang PJ, Teng RJ, Yau Kl: The human tail. Pediatr Neurol 19:230-233, 1998

10. McLone DG, Naidich TP: Terminal myelocystocele. Neurosurgery $16: 36-43,1985$

11. Samura K, Morioka T, Hashiguchi K, Yoshida F, Miyagi Y, Yoshiura T, Suzuki SO, Sasaki T: Coexistence of a human tail and congenital dermal sinus associated with lumbosacral lipoma. Childs Nerv Syst 25(1):137-141, 2009

12. Spiegelmann R, Schinder E, Mintz M, Blakstein A: The human tail: A benign stigma. J Neurosurg 63:461-462, 1985 